\title{
Novel metal allergy patch test using metal nanoballs
}

\author{
Tomoko Sugiyama ${ }^{1}$, Motohiro Uo ${ }^{2}$, Takahiro Wada ${ }^{2}$, Toshio Hongo ${ }^{2}$, Daisuke Omagari ${ }^{3}$, Kazuo Komiyama ${ }^{3}$, \\ Hitoshi Sasaki ${ }^{4}$, Heishichiro Takahashi ${ }^{5}$, Mikio Kusama ${ }^{1}$ and Yoshiyuki Mori ${ }^{1}$
}

\begin{abstract}
Background: Patch tests are often used in the clinical diagnosis of metal allergies. In currently available patch tests, high concentrations of metal salt solutions are used. However, diagnosis accuracy can be influenced not only by acute skin reactions to high concentrations of metal salt, but also by skin reactions to other components present in the patch or to $\mathrm{pH}$ changes. In this study, we developed Ni nanoparticles (termed "nanoballs") for use in patch-test solutions.
\end{abstract}

Findings: Highly soluble, spherical Ni nanoballs were prepared using plasma electrolysis. The Ni released from the nanoballs permeated through a dialysis membrane, and the nanoball-containing solution's pH was maintained constant. $\mathrm{Ni}$ ions were released slowly at low concentrations in a time-dependent manner, which contrasted the rapid release observed in the case of a commercial patch test. Consequently, in the new test system, reactions caused by high concentrations of metal salts were avoided.

Conclusions: By exploiting the high specific surface area of Ni nanoballs, we obtained an effective dissolution of $\mathrm{Ni}$ ions that triggered Ni allergy in the absence of direct contact between the nanoballs and mouse skin. This novel patch system can be applied to other metals and alloys for diagnosing various types of metal-induced contact dermatitis.

Keywords: Nanoparticle, Nickel, Metal allergy, Patch test, Elemental distribution

\section{Background}

Metal-allergy patch tests are routinely used in the clinical diagnosis of metal-induced contact dermatitis. Currently available patch tests use high concentrations of various metal salts in aqueous solution. They contain a reservoir sheet that allows the test solution to permeate into the skin to induce a local allergic reaction (Figure 1a). A patch test solution typically contains a metal salt under an acidic condition [1]. Current patch tests can cause pustular or follicular reactions because of the high concentration of metal salts. In addition, false positive or negative reactions [2] and skin irritation often occur [3-5]. Metal allergies, however, are often triggered by metal ions that are continuously eroded from metallic materials under neutral $\mathrm{pH}$ conditions, and in this case, the metal ion concentration is typically low. Factors such as $\mathrm{pH}$, metal-ion

\footnotetext{
* Correspondence: uo.abm@tmd.ac.jp

${ }^{2}$ Advanced Biomaterials Department, Graduate School of Medical and Dental Sciences, Tokyo Medical and Dental University, 1-5-45 Yushima, Bunkyo-ku, Tokyo 113-8549, Japan

Full list of author information is available at the end of the article
}

concentration, and dosage rates differ considerably between a genuine metal-allergic reaction and that which occurs in a patch test.

An ideal patch test must reproduce the metal erosion that occurs in metallic equipment (Figure 1b). Therefore, historically, large metal particles have been used as the metal source in the patch test [6]. However, their dissolution rates are inadequate for triggering an allergic reaction because of their low specific surface area $[7,8]$.

To address the aforementioned limitations of patch tests, in this study, we prepared 40-50-nm-diameter $\mathrm{Ni}$ nanoballs (Figure 1c) through plasma electrolysis at the surface of electrodes [9]. Because of their size, we expected the Ni nanoballs to exhibit a high rate of Ni dissolution, which is suitable for a patch test. In our novel patch system, we used a Ni-nanoball suspension enclosed in a dialysis tube, which we call a "Ni-nanoball pack." Ni ions released from the nanoball pack permeated through the dialysis membrane into the skin of mice, and thus direct contact between the Ni nanoballs and the skin was avoided. We evaluated this Ni-permeation behavior in vitro 


\section{(a) Current patch test}

(b) Ideal patch test

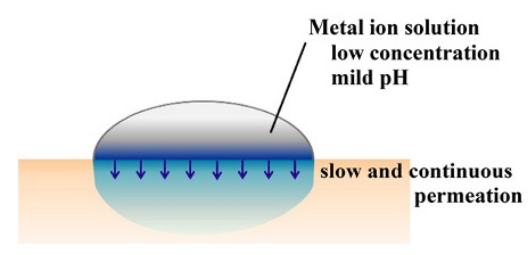

(c) Ni-nanoball patch test

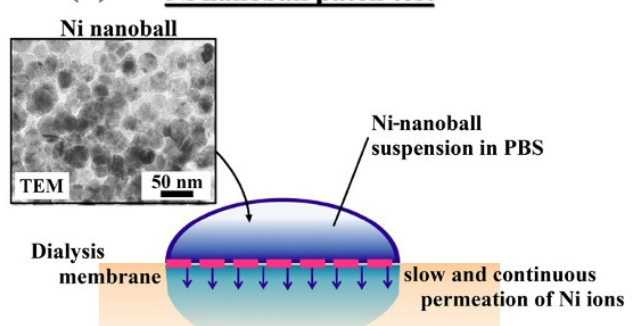

Figure 1 Various patch-test schemes. A currently available commercial patch test (a), an ideal patch test (b), and the novel patch test designed using Ni nanoballs (c).

and also in vivo by using mouse skin. The Ni distribution in the skin was measured using synchrotron radiationexcited X-ray fluorescence (SR-XRF) analysis and particleinduced X-ray emission (PIXE), which are highly sensitive analysis methods. Furthermore, we examined the chemical state of the Ni nanoballs and the permeated $\mathrm{Ni}$ in the skin by performing X-ray absorption fine structure (XAFS) analysis.

\section{Methods}

Preparation of Ni-nanoball suspensions and an in vitro test of $\mathrm{Ni}$-ion release

$\mathrm{Ni}$ nanoballs were synthesized according to the method described by Toriyabe et al. [9] Ni nanoballs were dispersed in distilled water (DW) and $0.1 \mathrm{M}$ phosphate buffer solution $(\mathrm{PBS})$ at $\mathrm{pH}=5.8$ to reach a concentration of $500 \mathrm{ppm}$ of $\mathrm{Ni}$ nanoballs. In the in vitro test of $\mathrm{Ni}$-ion release, the experimental setup used for examining $\mathrm{Ni}$ permeation through the dialysis membrane was prepared as shown in Figure $2 \mathrm{a}$. The inner cylinder was filled with $500 \mu \mathrm{L}$ of $\mathrm{Ni}$-nanoball suspension in DW and PBS $(\mathrm{pH}=5.8)$ and immersed in $2500 \mu \mathrm{L}$ of DW for $1 \mathrm{~h}$ to 7 days. Evaluation of the permeated $\mathrm{Ni}$ in the outer DW solution was based on a colorimetric reaction using 2-(5-Nitro-2pyridylazo)-5-[N-n-propyl-N-(3-sulfopropyl)amino]phenol disodium salt dehydrate (Nitro-PAPS), which results in the $\mathrm{Ni}$ complex and shows a characteristic absorption around $568 \mathrm{~nm}$ (Additional file 1: Figure S1). Then, $500 \mu \mathrm{L}$ of the outer solution was mixed with $2.0 \mathrm{~mL}$ of Nitro-PAPS aqueous solution $(20 \mathrm{ppm})$, and the $\mathrm{Ni}$ concentration was estimated by the absorbance at $568 \mathrm{~nm}$ using the optical absorption (UV-Vis) spectrometer based on Yamashita et al.
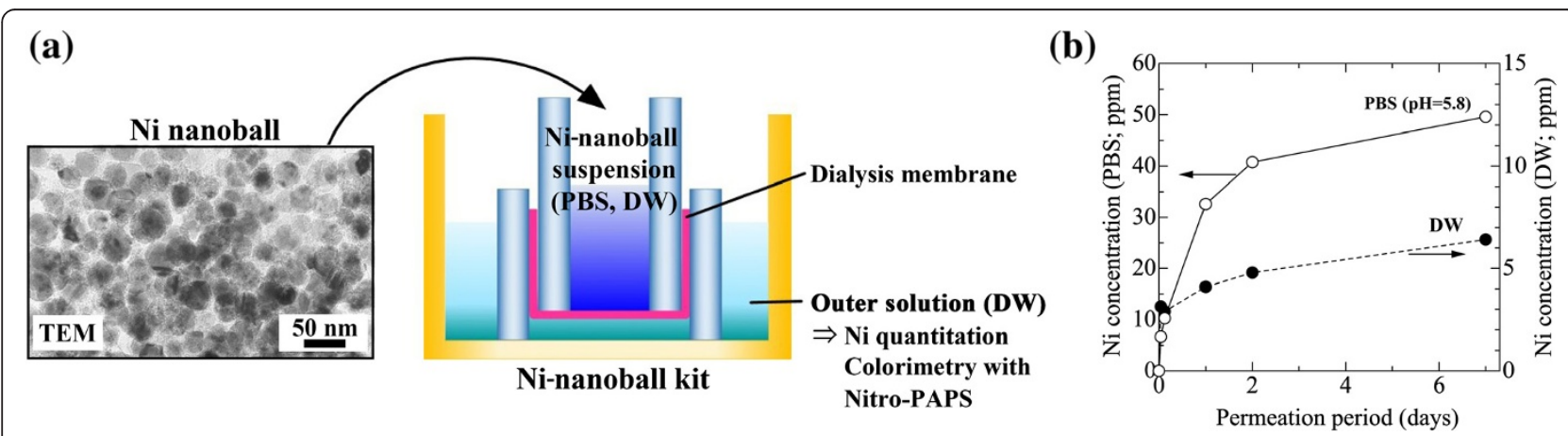

Figure 2 In vitro Ni release from Ni-nanoball suspensions through a dialysis membrane. Experimental setup (a) and time-dependent release of $\mathrm{Ni}(\mathbf{b})$. 
[10]. The standard Ni solutions (0.2-10 ppm) were prepared by diluting a $1 \mathrm{mg} / \mathrm{mL} \mathrm{Ni}\left(\mathrm{NO}_{3}\right)_{2}$ solution with $\mathrm{DW}$.

\section{In vivo $\mathrm{Ni}$-permeation test designed for mouse skin} C57BL/6 mice (45-75 weeks old) were used in this study. All animal protocols were approved by the Animal Ethics Review Board of the Dental Hospital of Nihon University School of Dentistry, Tokyo, Japan, and conformed to the guidelines of the National Institutes of Health. The skin on the back of mice was depilated under general anesthesia. Approximately $20 \mathrm{~mL}$ of the Ni-nanoball suspension was placed into the dialysis tube and both ends were clamped. The Ni-nanoball pack was fixed onto the depilated back skin of the mice by using film dressing. The application period ranged from $30 \mathrm{~min}$ to $24 \mathrm{~h}$, after which the mice were sacrificed. The Ni-nanoball pack was then removed and the skin was gently wiped, and the part of the skin that contacted the pack was excised and used for preparing frozen sections (20- $\mu \mathrm{m}$ thick) according to the method reported by Kubo et al. [11]. The sections were placed on Kapton film, dried and subjected to elemental-distribution analysis. As a control, a commercial patch test for $\mathrm{Ni}$ allergy (5\% w/v $\mathrm{NiSO}_{4}$ aq.) was applied to mice using the same method. Adjacent specimen slices were stained with hematoxylin and eosin (H-E) and used for histopathological diagnosis. Detailed information on the chemicals, materials, and equipments used are presented in Additional file 1: Table S1.

\section{Elemental-distribution and chemical-state analyses}

Elemental distribution analysis of the entire specimen was performed using SR-XRF. The specimen was irradiated with micro-focused X-ray and the specimen stage was scanned two- dimensionally. The fluorescence X-ray was detected at each point and the elemental distribution images were processed. The high-resolution elemental distribution was analyzed using micro-PIXE analysis, which involved exposure to the micro-focused proton beam with raster scanning over the target area of the specimen. The characteristic X-rays were detected, and the elemental distribution image was processed. The chemical states of the Ni contained in Ni nanoballs and the Ni in mouse skin were examined using XAFS analysis. Detailed conditions of the elemental analyses are shown in Additional file 1: Table S2.

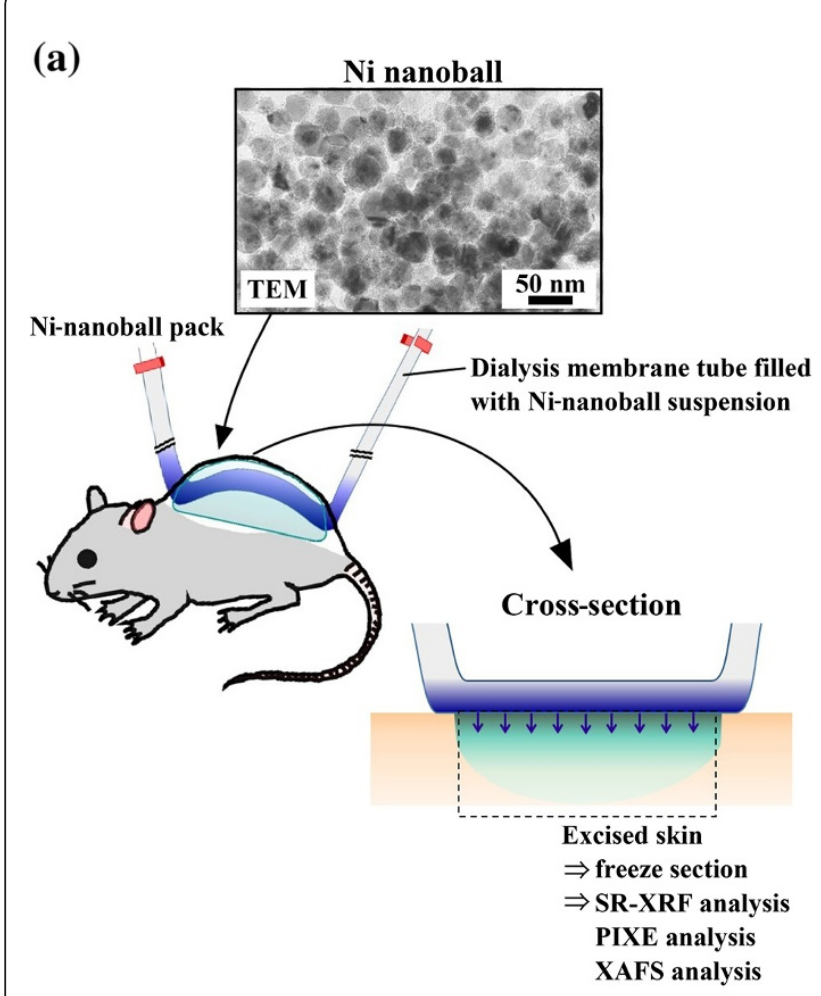

(b)
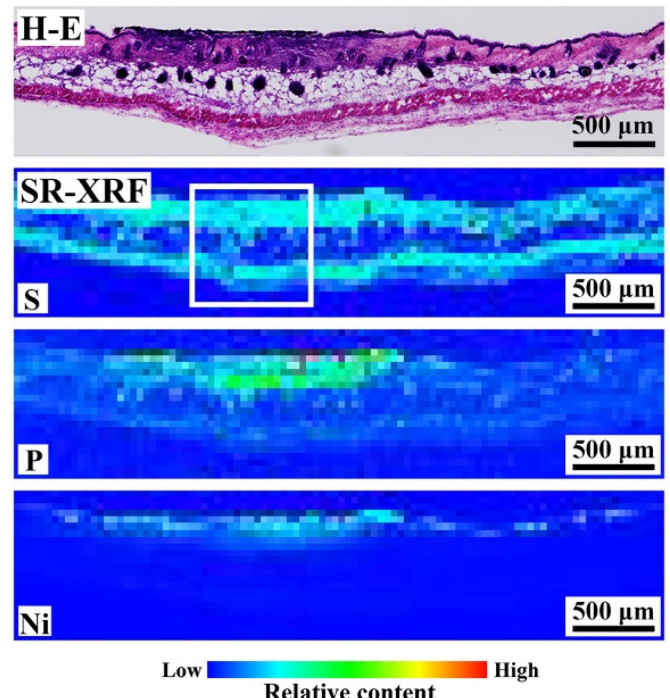

(c)

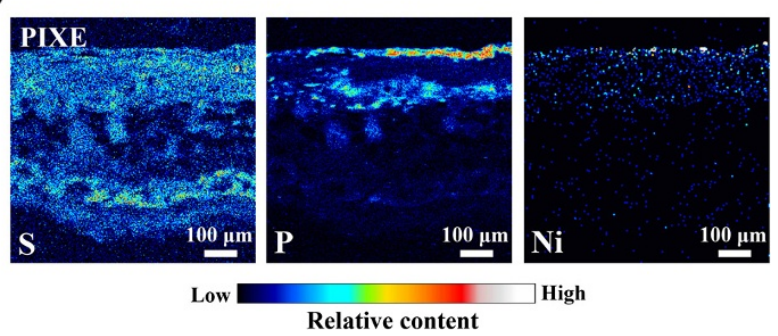

Figure 3 In vivo Ni permeation from Ni-nanoball packs into mouse skin after 24-h application. Experimental setup (a), histopathological (H-E) images and elemental-distribution images of skin cross-sections obtained using SR-XRF analysis (b), and detailed elemental-distribution images obtained using micro-PIXE analysis (c). 


\section{Results and discussion}

The time-dependent release of $\mathrm{Ni}$ in vitro is shown in Figure $2 \mathrm{~b}$. We observed a continuous release of Ni. The $\mathrm{Ni}$-dissolution rate of $\mathrm{Ni}$ nanoballs dispersed in PBS ( $\mathrm{pH}$ 5.8) was higher than that of nanoballs dispersed in DW. This result agrees with the previous findings of previous studies showing that $\mathrm{Ni}$ solubility is increased in acidic solutions $[12,13]$. The $\mathrm{pH}$ of the commercial Ni patch-test solution was 3.8, which is lower than that of natural human skin, where the $\mathrm{pH}$ is typically below 5.0 [14]. Thus, we do not expect the Ni-nanoball suspension at pH 5.8 to irritate human skin. Furthermore, the Ni-release rate in our test system could be controlled by adjusting the $\mathrm{pH}$.
Figure $3 \mathrm{~b}$ shows $\mathrm{H}$-E-stained cross-sections of mouse skin (histopathological analysis) and their SR-XRF images of elemental distribution obtained after treatment with the $\mathrm{Ni}$-nanoball pack for $24 \mathrm{~h}$. The Ni that permeated from the patch was clearly observed on the surface side of the skin. Figure 3c shows the detailed elemental distribution of $\mathrm{S}, \mathrm{P}$, and $\mathrm{Ni}$ in the areas that exhibited high $\mathrm{Ni}$ accumulation (white squares in Figure $3 \mathrm{~b}$ ), as assessed using microPIXE analysis. The Ni concentration was high in the epidermis and spread into the dermis layer beyond the basal layer. These results indicate a clear internal permeation of Ni. Furthermore, the H-E-stained images of the same area showed a slight inflammatory response at the

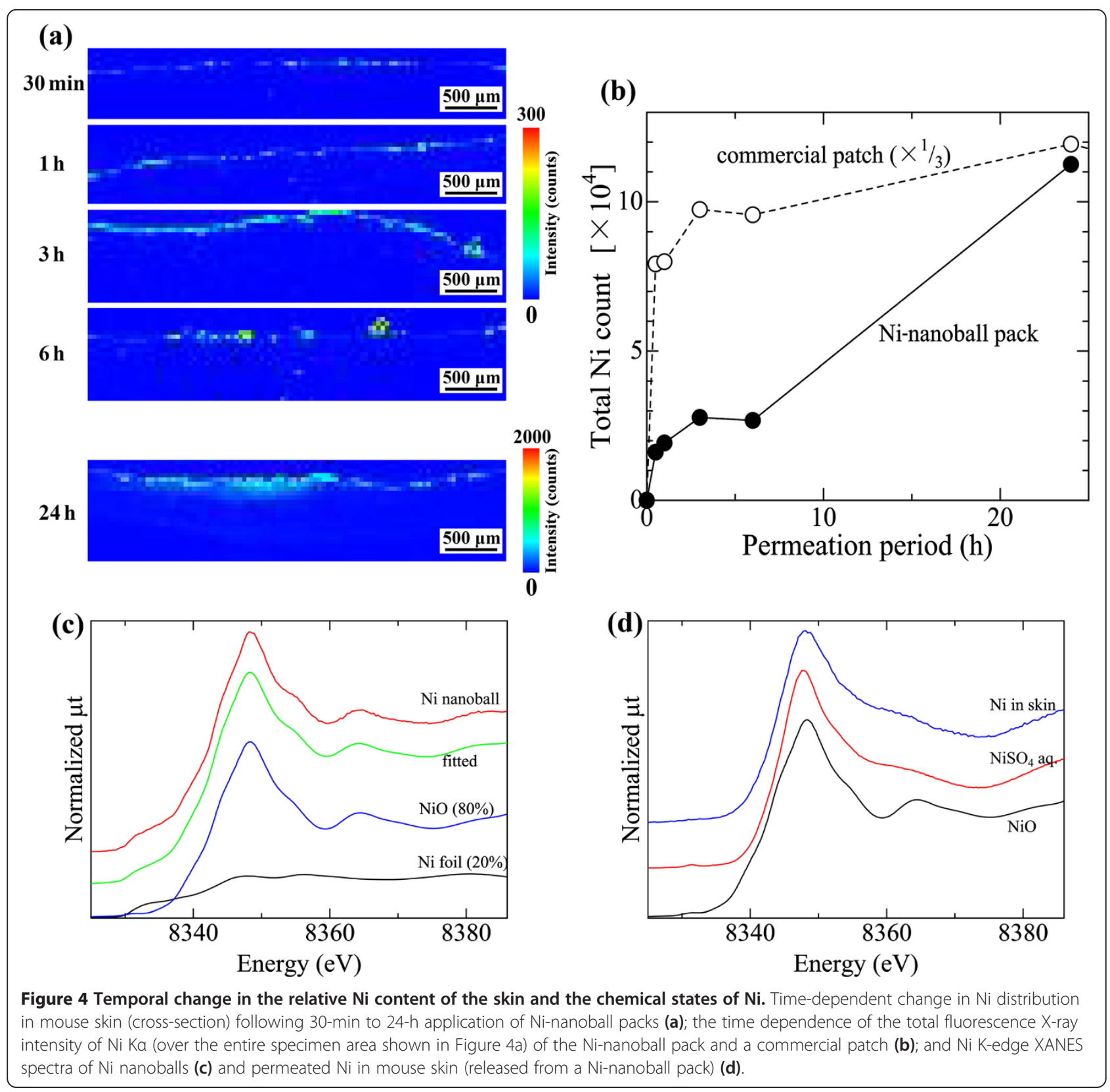


epidermis. The regions of inflammation overlapped with localized areas of $\mathrm{Ni}$ and $\mathrm{P}$ accumulation. Because the phosphate originates from inflammatory cells, this colocalization of $\mathrm{Ni}$ and $\mathrm{P}$ suggests that the Ni that permeates from the patch and penetrates the skin induces local inflammation.

Figure 4 shows the temporal change in the relative $\mathrm{Ni}$ content of the skin. Figure 4a shows the images of Ni distribution in the skin cross-section, and Figure $4 \mathrm{~b}$ shows the total fluorescence X-ray intensity of Ni Ko over the entire specimen area (shown in Figure 4a). When the $\mathrm{Ni}$-nanoball pack was used, the $\mathrm{Ni}$ content in the skin increased linearly in a time-dependent manner until $24 \mathrm{~h}$. Figure $4 \mathrm{~b}$ shows the result obtained when we applied the commercial Ni-allergy patch to the skin by using the same method. The concentration of the Ni that permeated from the commercial patch increased drastically after application for only $30 \mathrm{~min}$, and the permeated-Ni content was approximately three times higher than that after application for $24 \mathrm{~h}$. This extremely high dose of Ni is likely to lower the accuracy of the commercial patch test as a result of the potential side reactions that it might cause. By contrast, the comparatively slow and time-dependent permeation of $\mathrm{Ni}$ from the Ni-nanoball pack is likely to be optimal for eliciting a $\mathrm{Ni}$ allergic reaction.

Figure 4 also shows the Ni K-edge XAFS spectra of $\mathrm{Ni}$ nanoballs (Figure 4c) and the permeated $\mathrm{Ni}$ in skin (Ni released from the nanoballs) (Figure 4d). The spectrum of the Ni nanoballs closely fits that of a mixture of metallic $\mathrm{Ni}(20 \%)$ and $\mathrm{NiO}(80 \%)$. This suggests that the Ni nanoballs were almost oxidized during storage. Conversely, the spectrum of the permeated $\mathrm{Ni}$ in skin (derived from the $\mathrm{Ni}$-nanoball pack) was similar to that of $\mathrm{NiSO}_{4}$ aq., but distinct from that of $\mathrm{NiO}$, a major component of $\mathrm{Ni}$ nanoballs. Therefore, the dissolved $\mathrm{Ni}$ ions were successfully released from the Ni nanoballs and they permeated the skin through the dialysis membrane.

During a genuine allergic reaction to metals, metal erosion and skin permeation occur in a low and continuous dose. We have shown that our Ni-nanoball pack releases $\mathrm{Ni}$ ions slowly from the suspension under mildly acidic conditions. Recently, the health risks posed by nanomaterials have become a growing concern [15-18]. Thus, in this study, we exploited the high specific surface area of $\mathrm{Ni}$ nanoballs in order to obtain an effective dissolution of $\mathrm{Ni}$ ions and used this to trigger $\mathrm{Ni}$ allergy without direct contact between the nanoballs and the skin.

\section{Conclusions}

A high dose of metals and counter-ions and an acidic $\mathrm{pH}$ are factors that lower the accuracy of currently available metal-allergy patch tests. These factors were addressed effectively in this study, and the test system presented here could potentially serve as a state-of-the-art patch test. Another limitation of current patch tests is that they are available only for a few metal species, and the accuracy of these tests is still in question. The facile process used in this study for preparing $\mathrm{Ni}$ nanoballs can be applied to most pure metals and alloys, and then the method can be readily used for diagnosing other metal allergies. By conducting further studies on the ability to induce allergy in animal and human skin, the accuracy of the diagnosis obtained using our novel patch test designed for metal allergy could be potentially enhanced.

\section{Additional file}

Additional file 1: Table S1. Detailed information on chemicals, materials, and equipment. Table S2. Detailed experimental conditions used for elemental analysis. Figure S1. Optical absorption spectra of Nitro-PAPS solution mixed with $\mathrm{Ni}$ ion solutions of various concentrations (a) and the calibration curve of $\mathrm{Ni}$ with the absorbance at $568 \mathrm{~nm}$ (b). Linear correlation was observed between 0 to $2.0 \mathrm{ppm}$ of Ni. Quantitation of $\mathrm{Ni}$ was carried within this range by diluting the sample solution adequately.

\section{Abbreviations}

SR-XRF: Synchrotron radiation-excited X-ray fluorescence; PIXE: Particle-induced X-ray emission; XAFS: X-ray absorption fine structure; PBS: Phosphate buffer solution; Nitro-PAPS: 2-(5-Nitro-2-pyridylazo)-5-[N-n-propyl-N-(3-sulfopropyl)amino]phenol disodium salt dehydrate.

\section{Competing interests}

The authors declare that they have no competing interests.

\section{Authors' contributions}

TS and MU conceived and designed the experiments. HS and HT produced and measured the Ni nanoballs. TS, MU, and DO performed the experiments. DO and KK performed the histopathological analysis. TS, MU, and TW performed the SR-XRF and PIXE analyses. TS and MU co-wrote the manuscript. KK, MK, and YM edited the manuscript. All authors discussed the results and commented on the manuscript. All authors read and approved the final manuscript.

\section{Acknowledgements}

The SR-XRF measurements were performed with the approval of the Photon Factory Program Advisory Committee (Proposal Nos. $2011 \mathrm{G} 011$ and 2014G017). This work was financially supported by the Japan Society for the Promotion of Science KAKENHI (Grant No. 23390438 to M. Uo). T. Sugiyama is supported by a research fellowship for young scientists from the Japan Society for the Promotion of Science.

\section{Author details}

${ }^{1}$ Department of Dentistry, Oral and Maxillofacial Surgery, Jichi Medical University, 3311-1 Yakushiji, Shimotsuke, Tochigi 329-0498, Japan. ${ }^{2}$ Advanced Biomaterials Department, Graduate School of Medical and Dental Sciences, Tokyo Medical and Dental University, 1-5-45 Yushima, Bunkyo-ku, Tokyo 113-8549, Japan. ${ }^{3}$ Department of Pathology, Nihon University School of Dentistry, 1-8-13 Kanda-Surugadai, Chiyoda-ku, Tokyo 131-8310, Japan. ${ }^{4}$ Nakayamagumi Co. Ltd., North 19, East 1, Higashi-ku, Sapporo, Hokkaido 065-8610, Japan. ${ }^{5}$ Graduate School of Engineering, Hokkaido University, North 13, West 8, Sapporo, Hokkaido 060-8628, Japan.

Received: 15 September 2014 Accepted: 6 November 2014 Published online: 03 December 2014

\section{References}

1. Endo K, Ohno H, Kawashima I, Yamane Y, Yanagi T: Reliability of patch testing for metal hypersensitivity: Stability of metal ions and solution pH. Shika Zairyo Kikai 2005, 24:82.

2. Mitchell J, Maibach HI: Managing the excited skin syndrome: patch testing hyperirritable skin. Contact Dermatitis 1997, 37:193-199. 
3. Mowad CM: Patch testing: pitfalls and performance. Curr Opin Allergy Clin Immunol 2006, 6:340-344.

4. Diepgen TL, Coenraads PJ: Sensitivity, specificity and positive predictive value of patch testing: the more you test, the more you get? ESCD Working Party on Epidemiology. Contact Dermatitis 2000, 42:315-317.

5. Fischer T, Rystedt I: False-positive, follicular and irritant patch test reactions to metal salts. Contact Dermatitis 1985, 12:93-98.

6. Thyssen JP, Menné T, Schalock PC, Taylor JS, Maibach HI: Pragmatic approach to the clinical work-up of patients with putative allergic disease to metallic orthopaedic implants before and after surgery. Br J Dermatol 2011, 164:473-478.

7. Midander K, Pan J, Wallinder IO, Heim K, Leygraf C: Nickel release from nickel particles in artificial sweat. Contact Dermatitis 2007, 56:325-330.

8. Larese F, Gianpietro A, Venier M, Maina G, Renzi N: In vitro percutaneous absorption of metal compounds. Toxicol Lett 2007, 170:49-56.

9. Toriyabe $Y$, Watanabe $S$, Yatsu S, Shibayama T, Mizuno T: Controlled formation of metallic nanoballs during plasma electrolysis. Appl Phys Lett 2007, 91:041501.

10. Yamashita S, Abe A, Noma A: Sensitive, direct procedures for simultaneous determinations of iron and copper in serum, with use of 2-(5-nitro-2pyridylazo)-5-(N-propyl-N-sulfopropylamino)phenol (nitro-PAPS) as ligand. Clin Chem 1992, 38:1373-1375.

11. Kubo A, Ishizaki I, Kubo A, Kawasaki H, Nagao K, Ohashi Y, Amagai M: The stratum corneum comprises three layers with distinct metal-ion barrier properties. Sci Rep 2013, 3:1731. doi:10.1038/srep01731.

12. Palmer DA, Bénézeth $P$, Xiao C, Wesolowski DJ, Anovitz LM: Solubility measurements of crystalline $\mathrm{NiO}$ in aqueous solution as a function of temperature and pH. J Solution Chem 2011, 40:680-702.

13. Plyasunova NV, Zhang Y, Muhammed M: Critical evaluation of thermodynamics of complex formation of metal ions in aqueous solutions. IV. Hydrolysis and hydroxo-complexes of $\mathrm{Ni}^{2+}$ at $298.15 \mathrm{~K}$. Hydrometallurgy 1998, 48:43-63.

14. Lambers $H$, Piessens $S$, Bloem $A$, Pronk $H$, Finkel P: Natural skin surface $\mathrm{pH}$ is on average below 5 , which is beneficial for its resident flora. Int I Cosmetic Sci 2006, 28:359-370.

15. Auffan M, Rose J, Bottero JY, Lowry GV, Jolivet JP, Wiesner MR: Towards a definition of inorganic nanoparticles from an environmental, health and safety perspective. Nat Nanotechnol 2009, 4:634-641.

16. Kertész Z, Szikszai Z, Gontier E, Moretto P, Surlève-Bazeille JE, Kiss B, Juhász I, Hunyadi J, Kiss ÁZ: Nuclear microprobe study of $\mathrm{TiO}_{2}$-penetration in the epidermis of human skin xenografts. Nucl Instrum Methods Phys Res, Sect $B$ Beam Interact with Mater Atoms 2005, 231:280-285.

17. Larese Filon F, Crosera M, Timeus E, Adami G, Bovenzi M, Ponti J, Maina G: Human skin penetration of cobalt nanoparticles through intact and damaged skin. Toxicol In Vitro 2013, 27:121-127.

18. Larese FF, D'Agostin F, Crosera M, Adami G, Renzi N, Bovenzi M, Maina G: Human skin penetration of silver nanoparticles through intact and damaged skin. Toxicology 2009, 255:33-37.

doi:10.1186/s12951-014-0051-7

Cite this article as: Sugiyama et al:: Novel metal allergy patch test using metal nanoballs. Journal of Nanobiotechnology 2014 12:51.

\section{Submit your next manuscript to BioMed Central and take full advantage of:}

- Convenient online submission

- Thorough peer review

- No space constraints or color figure charges

- Immediate publication on acceptance

- Inclusion in PubMed, CAS, Scopus and Google Scholar

- Research which is freely available for redistribution

Submit your manuscript at www.biomedcentral.com/submit
C Biomed Central 